\title{
Effects of human papillomavirus (HPV) type 16 oncoproteins on the expression of involucrin in human keratinocytes
}

\author{
Eszter Gyöngyösi, Anita Szalmás, Annamária Ferenczi, József Kónya, Lajos Gergely and György Veress
}

\begin{abstract}
Background: The human papillomavirus (HPV) life cycle is closely linked to keratinocyte differentiation. Oncogenic HPV infection has been shown to hamper the normal differentiation of keratinocytes; however, the underlying mechanisms responsible for this phenomenon are yet to be clarified. Here, we aimed to study the effects of HPV16 E6 and E7 oncogenes on the expression of involucrin (IVL), an established marker of keratinocyte differentiation, in human foreskin keratinocyte (HFK) cells.

Results: The differentiation of HFK cells by serum and high calcium significantly increased both the mRNA and the protein levels of IVL. The E6 and E7 oncoproteins of HPV16 together caused strong down-regulation of IVL mRNA and protein both in proliferating and in differentiating HFK cells. To study the effects of HPV oncogenes on the IVL promoter, we made transient transfection assays and luciferase tests and found that HPV 16 E6 but not E7 repressed IVL promoter activity in proliferating HFK cells. The inhibitory effect of HPV 16 E6 on the human IVL promoter could be localised to the proximal regulatory region (PRR) of the gene.

Conclusions: These results suggest that the down-regulation of IVL promoter activity by HPV 16 E6 significantly contribute to the inhibition of endogenous IVL expression by the HPV 16 oncoproteins. In contrast, the downregulation of endogenous IVL expression by HPV16 E7 is probably not caused by a direct and specific effect of E7 on the IVL promoter.
\end{abstract}

Keywords: HPV 16, Oncogenes, Keratinocyte differentiation, Involucrin

\section{Background}

Papillomaviruses are small DNA viruses, with a circular double-stranded DNA genome of about $8 \mathrm{kbp}$ length [1]. Over 100 human papillomavirus (HPV) types have been identified until now, of which about 40 is able to infect the genital mucosa [2]. Low-risk HPV types (HPV $6,11,42$ ) are mainly found in benign genital lesions (condyloma acuminatum) or low grade cervical dysplasias, while high-risk or oncogenic genital types (HPV 16, 18 , and others) are causally linked to the development of cervical cancer [3].

The E6 and E7 oncoproteins of high-risk HPVs are responsible for the transforming activity of the virus [4]. High-risk HPV E6 induces the degradation of the p53

\footnotetext{
* Correspondence: veregy@med.unideb.hu

Department of Medical Microbiology, Medical and Health Science Centre, University of Debrecen, 4032 Debrecen, Nagyerdei krt. 98, Hungary
}

tumour suppressor protein through the ubiquitin-proteosome pathway [5]. In addition, HPV E6 is able to bind several other cellular proteins, some of which can mediate transforming activity independently from the p53 pathway [5]. High-risk HPV E7 is able to bind to the pRB (retinoblastoma) tumour suppressor protein, resulting in the functional inactivation and degradation of pRB [6]. By binding to pRB/E2F complex and, by releasing free E2F transcription factors, HPV E7 induces the progression of the cell cycle [6].

The life cycle of human papillomaviruses is closely linked to keratinocyte differentiation. HPVs initially infect proliferating basal cells of the squamous epithelium, while virus production is associated with terminally differentiated layers [7]. The cellular DNA replication machinery is reactivated by the E7 oncogene in differentiating keratinocytes to provide a cellular environment
C Biomed Central 
that is permissive for the replication of the viral genome $[7,8]$. This activity of HPV 16 E7 was shown to delay the induction of the keratinocyte differentiation markers involucrin and keratin 10 [9].

During the multi-step process of keratinocyte differentiation, the expression of genes involved in the process (such as keratins, transglutaminase 1, involucrin, etc.) is tightly regulated. The presence of HPV 16 E6 oncogene was shown to hamper the normal differentiation of keratinocytes induced by serum and calcium or by normal stratification in organotypic cell culture [10-12]. However, the underlying mechanisms responsible for the perturbed differentiation of keratinocytes by the HPV oncogenes are only partially elucidated.

It is reasonable to assume that the E6 and/or E7 oncogenes may have an effect on the transcription of key cellular genes involved in the differentiation of squamous epithelium. Indeed, HPV 16 E6 was shown to modulate the expression of several differentiation-associated genes in human foreskin keratinocytes [13]. Using a xenograft model, Lehr and co-workers showed that infection of human keratinocytes by certain HPVs (type 11 and 59) causes altered expression of certain CCE (cornified cell envelope) proteins, such as loricrin and small proline rich proteins (SPRR), both on the mRNA and on the protein level $[14,15]$. However, it is not known whether the HPV oncoproteins have effects on the promoters of differentiation-regulated genes or exert their effects post-transcriptionally.

The aim of this study was to investigate the effects of HPV 16 E6 and E7 oncogenes on the expression of the involucrin (IVL) and transglutaminase 1 (TG1) genes, which are established markers of differentiation of squamous epithelium. IVL protein is a $68 \mathrm{kDa}$, rod-shaped molecule containing several glutamine residues. It is found in the cytoplasm and cross-linked to membrane proteins by keratinocyte transglutaminases in differentiating keratinocytes [16].

Here, we found that the HPV oncogenes down-regulated both $I V L$ mRNA and protein levels in human foreskin keratinocyte (HFK) cells. In order to study the molecular mechanisms that are responsible for the gene expression alterations by the HPV oncoproteins, experiments were performed using HPV 16 E6 and/or E7 expression plasmids along with luciferase reporter constructs containing parts of the regulatory region of the human $I V L$ gene.

\section{Results}

Generation and characterization of human foreskin keratinocyte (HFK) cells expressing HPV 16 oncogenes

To study the effects of HPV oncogenes on the expression of cellular genes, human foreskin keratinocyte (HFK) cells were transduced by recombinant retroviruses carrying either the control vector (LXSN) or vectors encoding HPV16 E6 or E7 or both oncogenes. The presence of HPV E6 and/or E7 mRNA in the transduced cells was demonstrated by RT-PCR (Figure 1A). The 2 PCR bands shown on Figure 1A (upper panel) represent the full length E6 mRNA and the E6*I spliced variant, in accordance with previous results [17]. The presence of functionally active HPV oncoproteins was confirmed by demonstrating their effect on the level of the cellular p53 oncoprotein (Figure 1B). As expected, transduction of HPV16 E6 resulted in a decreased level of p53 protein (about $10 \%$ of that seen in control transduced cells, as indicated by densitometry), while expression of HPV 16 E7 led to the stabilization and thus higher level (1.6-fold increase) of p53 protein [5,6].

The effects of the HPV oncogenes on the endogenous mRNA levels of different cellular genes were studied using real-time RT-PCR assays. In these experiments, cell lines were used within 5 to 6 passages after transduction, as we wanted to see the acute effects of the HPV oncogenes on the expression of the cellular genes. The transduced cells maintained the proliferating, non-differentiating phenotype when left in serum-free medium, while differentiation was induced by changing the culture medium to DMEM (with serum and high calcium) for $24 \mathrm{~h}$, in accordance with the results of previous studies [9,11]. An established function of the HPV 16 E6 oncogene is the transcriptional trans-activation of the human TERT (telomerase reverse transcriptase) promoter $[18,19]$. Accordingly, the presence of E6 led to a considerable (about 300-fold) increase in the level of TERT mRNA $(p<0.001)$, while E7 had a weaker but still significant $(p<0.05)$ effect (Figure 2A). As expected, induction of differentiation had no significant effect on the level of TERT mRNA in any of the transduced cells.

\section{Effects of HPV 16 oncogenes on the expression of selected cellular genes involved in keratinocyte differentiation}

Real-time RT-PCR assays were used to examine the effects of the HPV oncogenes on the expression of the squamous differentiation marker transglutaminase 1 (TG1) and one of its major substrate involucrin $(I V L)$ in HPV oncogene transduced cells. As expected, induction of differentiation of HFK cells by serum and increased calcium resulted in highly increased levels of both $I V L$ and TG1 mRNA $(p<0.005)$ (Figure 2B and 2C). In proliferating cells, both E6 and E7 had significant inhibiting effect $(p<0.01)$ on IVL mRNA levels (Figure 2B). We found a very strong down-regulation of $I V L$ mRNA in cells expressing both HPV oncoproteins (HFK-16E6E7) compared to vector transduced (LXSN) cells $(p<0.001)$. Both E6 and E7 caused significant $(p<0.001)$ down-regulation of $I V L$ mRNA in differentiating HFK cells. 
(A)
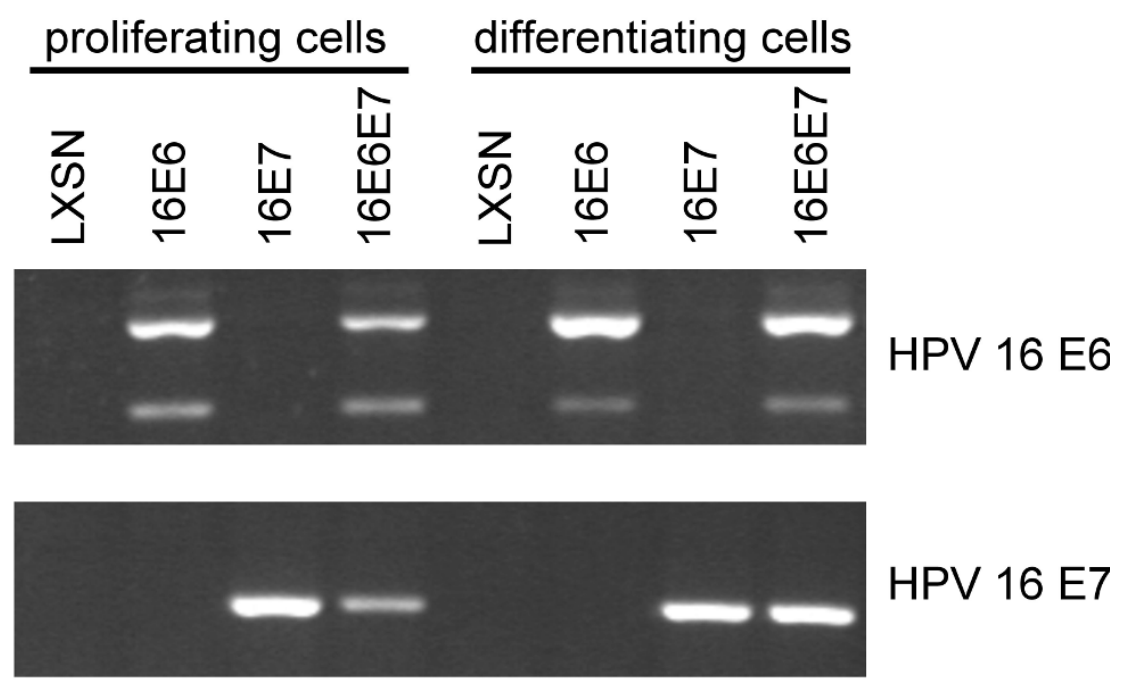

HPV 16 E7

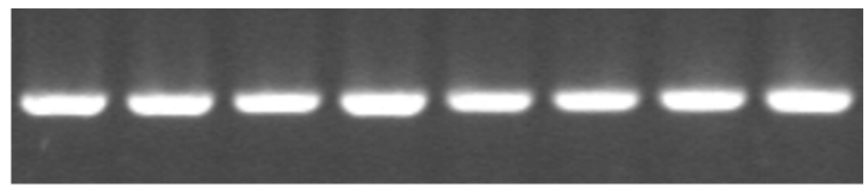

GAPDH

(B)

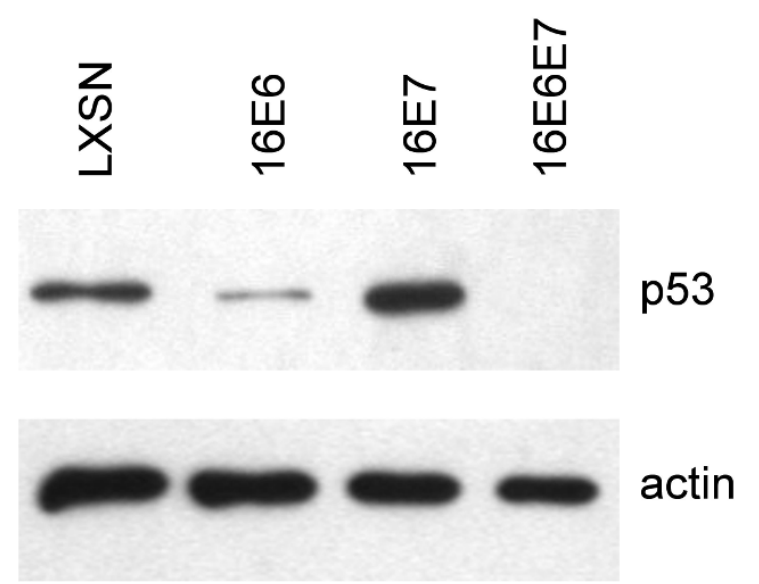

Figure 1 The expression of HPV oncogenes in transduced human foreskin keratinocyte (HFK) cells. (A) Cells were transduced by recombinant retroviruses carrying either the control vector (LXSN) or vectors encoding HPV16 E6, E7, or E6/E7. The cells were either left in serum-free medium (proliferating cells) or induced to differentiate in DMEM (with serum and high calcium). RT-PCR analysis was used to confirm the expression of the appropriate HPV oncogenes in the different cell lines (GAPDH was used as an endogenous control). (B) The effect of HPV16 E6 and E7 on the level of p53 protein. Protein extracts from transduced cells were subjected to Western blot assay for p53 or actin (the latter as loading control). Parts of representative X-ray films are shown.

Again, the strongest effect was seen in cells expressing E6 and E7 together $(p<0.001)$. In proliferating cells, HPV 16 E6 and E7 oncogenes together had only moderate inhibiting effect on the endogenous mRNA level of TG1 $(p<0.05)$, while in differentiating cells, the HPV oncogenes had no significant effect on TG1 

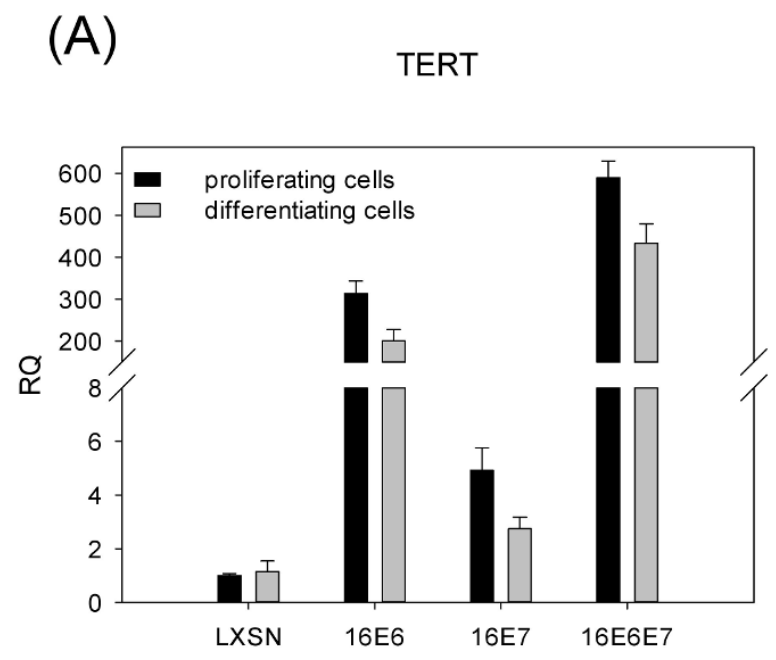

(B)

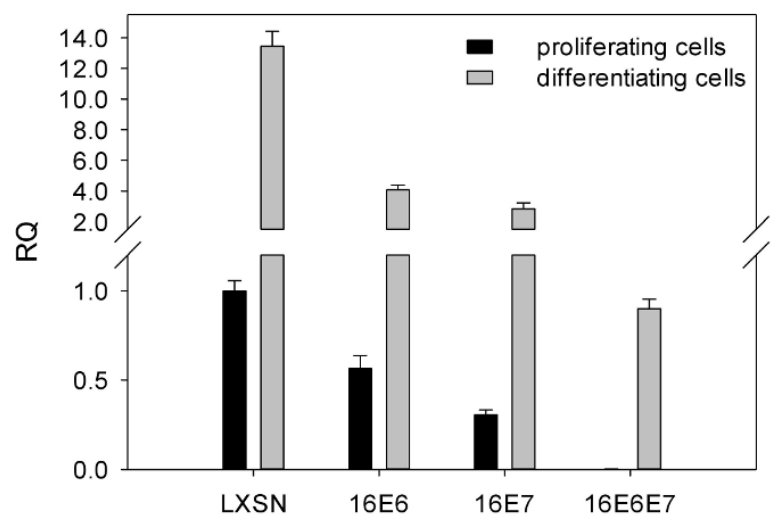

(C)

TG1

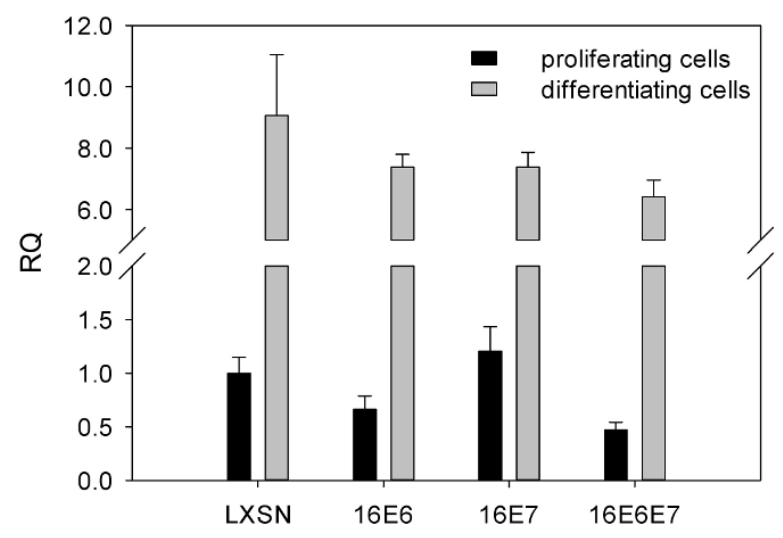

Figure 2 The effect of HPV oncogenes on the mRNA levels of selected genes in HFK cells. Generation of cell lines and induction of differentiation was performed as described in the legend to Figure 1. Relative Quantification (RQ) values were obtained by real-time RT-PCR analysis to see the effects of HPV 16 oncogenes on the level of TERT (A), IVL (B), or TG1 (C) MRNA in HFK cells. The RQ value of proliferating cells transduced by LXSN was set to 1, and other values are shown relative to this. Each reaction was performed in triplicate at least three times. Representative graphs are shown, with error bars indicating standard deviation. 
mRNA expression (Figure $2 \mathrm{C}$ ). These results suggest that the effect of the HPV oncogenes on IVL expression is either more direct or more specific than that on TG1 expression.

To see the effects of the HPV oncogenes on the level of the IVL protein, we performed Western blot analysis using monoclonal antibody to IVL (Figure 3). Induction of differentiation by serum and high calcium resulted in increased IVL protein levels, as compared to that seen in proliferating cells. In proliferating cells, HPV 16 E6 or E7 alone had little effect on endogenous IVL protein, while the two oncogenes together caused strong down-

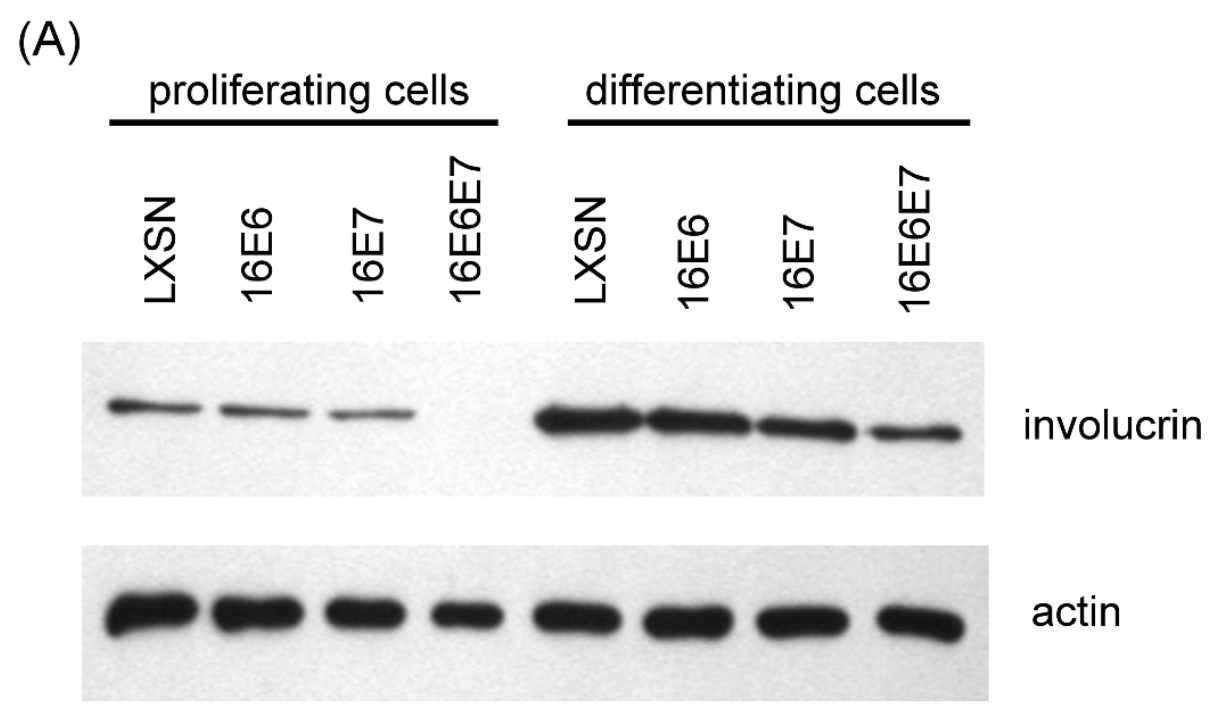

(B)

Densitometry

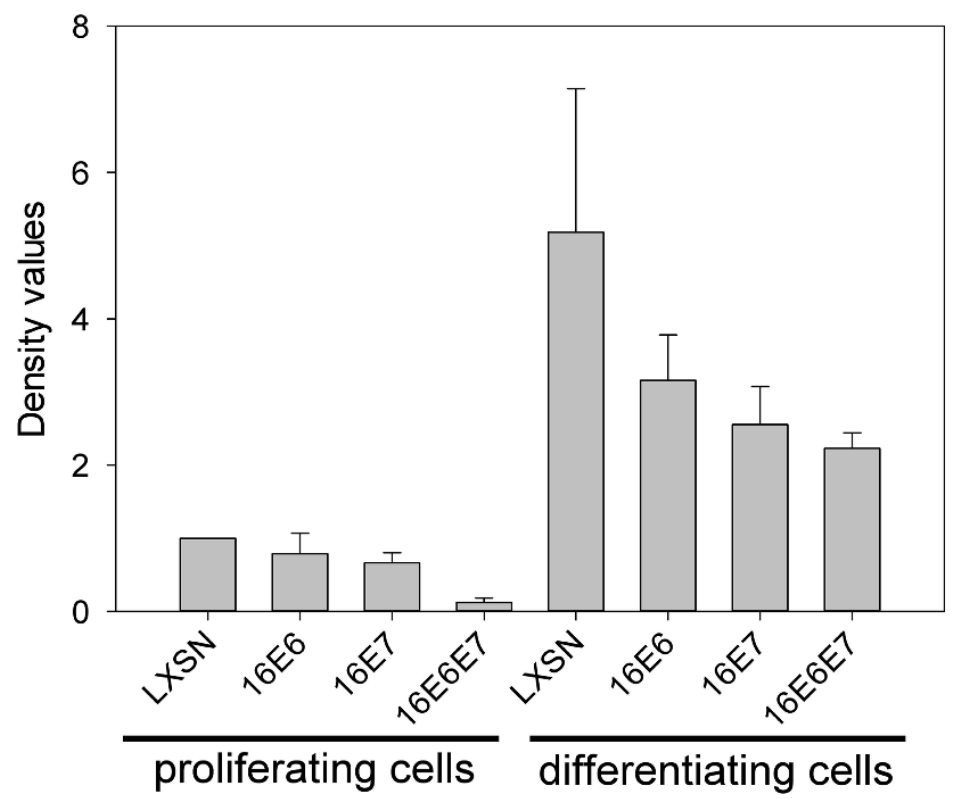

Figure 3 The effect of HPV oncogenes on the level of IVL protein in HFK cells. Generation of cell lines and induction of differentiation was performed as described in the legend to Figure 1. Ten $\mu \mathrm{g}$ of protein extracts prepared from the indicated cell lines were subjected to Western blot analysis for IVL or actin protein (the latter as loading control). (A) Parts of representative X-ray films are shown. (B) The amount of IVL protein was quantitatively analyzed by densitometry and standardized to actin protein level. The standardized density value of cycling cells transduced by LXSN was set to 1, and other values are shown relative to this. Values show the means from four independent experiments with standard errors shown as error bars. 
regulation of IVL protein level as compared to that found in vector transduced (LXSN) cells $(p<0.001)$. This down-regulating effect of E6/E7 on IVL protein level was smaller but still significant in differentiating cells $(p=0.032)$.

\section{Effects of HPV 16 oncogenes on the transcriptional activity of the human IVL promoter}

Next, we aimed to determine whether the down-regulation of $I V L$ expression by the HPV oncoproteins was caused by inhibiting the transcriptional activity of the $I V L$ promoter. To this end, low-passage HFK cells were transiently transfected by either vector control or HPV 16 E6 and/or E7 expression vectors along with luciferase reporter constructs containing the regulatory region of the human $I V L$ gene. In pilot experiments, we used an internal control vector (a Renilla luciferase reporter vector) to standardize for transfection efficiency. However, we found that HPV 16 E6 expression had an effect on the activity of the internal control vector. Therefore, here we show luciferase results obtained by standardization for protein concentration. The expression of functionally active HPV 16 E6 and E7 proteins in the transfected HFK cells was confirmed by co-transfection of reporter constructs containing either binding sites for the $\mathrm{p} 53$ protein or the adenovirus E2 (AdE2) promoter carrying binding site for the E2F transcription factor. As expected, the expression of HPV 16 E6 caused the down-regulation of the p53 reporter construct in proliferating HFK cells (Figure 4A), while HPV 16 E7 increased the activity of the adenovirus E2 promoter (Figure $4 \mathrm{~B})$.

In proliferating HFK cells, HPV 16 E6 caused significant down-regulation of a reporter construct (pGL3-IVL) containing the full-length regulatory region of the human $I V L$ gene $(p<0.001)$. On the contrary, E7 alone had no significant effect $(p=0.485)$ on the $I V L$ reporter construct in proliferating cells (Figure $4 \mathrm{C}$ ). Induction of differentiation by serum and high calcium resulted in highly (15-fold) increased activity of the pGL3-IVL construct in the presence of the empty expression vector pcDNA. In differentiating HFK cells, both HPV 16 E6 $(p=0.124)$ and E7 ( $p=$ 0.253 ) had a non-significant tendency to inhibit $I V L$ promoter activity, while HPV 16 E6 and E7 together caused significant down-regulation of the $I V L$ promoter ( $p=$ 0.014) (Figure $4 C$ ). Taken together, these results indicate that the down-regulation of IVL promoter activity by HPV 16 E6 significantly contribute to the inhibition of endogenous IVL expression by the HPV 16 oncoproteins.

\section{Localisation of the effects of HPV 16 oncogenes on the human IVL promoter}

In order to localise the effects of HPV 16 oncogenes on the human $I V L$ promoter, a series of luciferase reporter vectors was constructed carrying different fragments of the promoter all extending to $n t+41$ relative to the transcriptional start site (Figure 5A). First, we checked in transient transfection experiments whether these constructs responded to differentiation stimuli in HFK cells. As shown on Figure 5B, the activity of each reporter construct was induced by differentiation of HFK cells. Induction by differentiation was highest (15-fold) for the longest construct (IVL-2418), while it was lower (about 4-fold) for the shorter $I V L$ promoter constructs. Next, we studied the effects of HPV 16 E6 and E7 on the transcriptional activity of the IVL reporter constructs in HFK cells. In proliferating HFK cells, HPV 16 E6 was able to down-regulate transcription from each reporter construct. When compared to vector control transfected cells, E6 reduced the activity of the full-length reporter construct IVL-2418 $(p=0.026)$ and also that of the shortest reporter construct IVL-272 ( $p=0.011)$ containing only the PRR (proximal regulatory region) of the IVL promoter (Figure 5C). E6 caused significant downregulation of all the tested IVL reporter constructs also in differentiating HFK cells (Figure 5D). These results indicate that most of the down-regulating activity of HPV 16 E6 on the human $I V L$ promoter can be mapped to the proximal regulatory region of the $I V L$ gene (to the promoter region -231 to +41 relative to the transcriptional start site).

HPV 16 E7 had no significant effect on any IVL reporter construct in proliferating cells (Figure 5C). In differentiating cells, E7 had a moderate but significant inhibiting effect $(p=0.020)$ on the full-length IVL reporter construct (IVL-2418), while it had no significant effect on the shorter IVL reporter constructs.

\section{Discussion}

In this study, we found that the HPV 16 E6 and E7 oncoproteins caused a synergistic down-regulation of endogenous IVL mRNA and protein levels in HFK cells, which are natural host cells of the virus. Our finding is in accordance with previous studies performing microarray analysis of genes involved in cervical carcinogenesis. IVL and/or other keratinocyte differentiation associated genes (such as certain keratins and small prolin-rich proteins) are down-regulated in cervical cancer specimens compared to normal cervical samples [20,21]. Accordingly, several studies using cultured human keratinocytes as in vitro models for cervical carcinogenesis found that the expression of HPV oncogenes causes a down-regulation of expression of $I V L$ and/or other genes involved in epithelial differentiation $[13,22,23]$. However, microarray analysis does not provide information on the mechanism of changes in gene expression. Therefore, our approach was to analyse in HFK cells gene expression alterations of a few selected genes involved in keratinocyte differentiation using reliable real-time RT-PCR assays and to 


\section{(A) \\ p53 reporter}

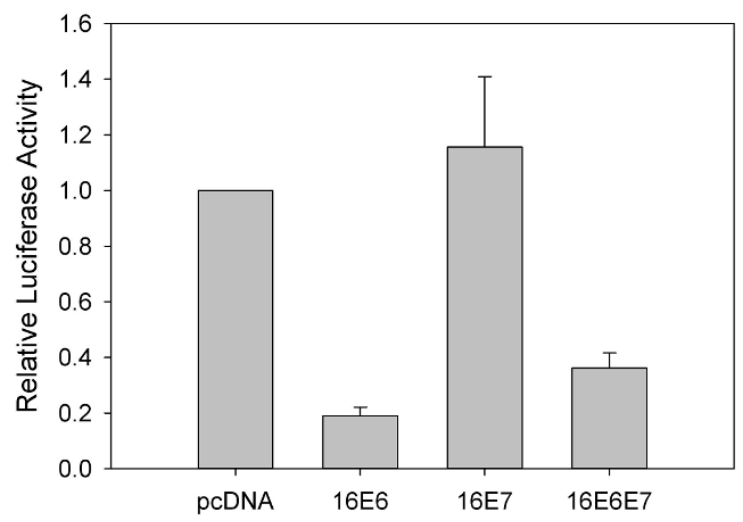

(B)

Adenovirus E2 reporter

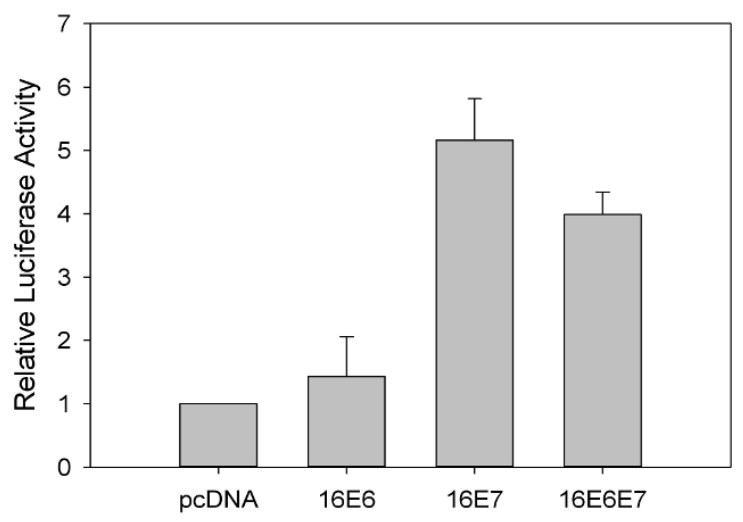

(C)

IVL reporter

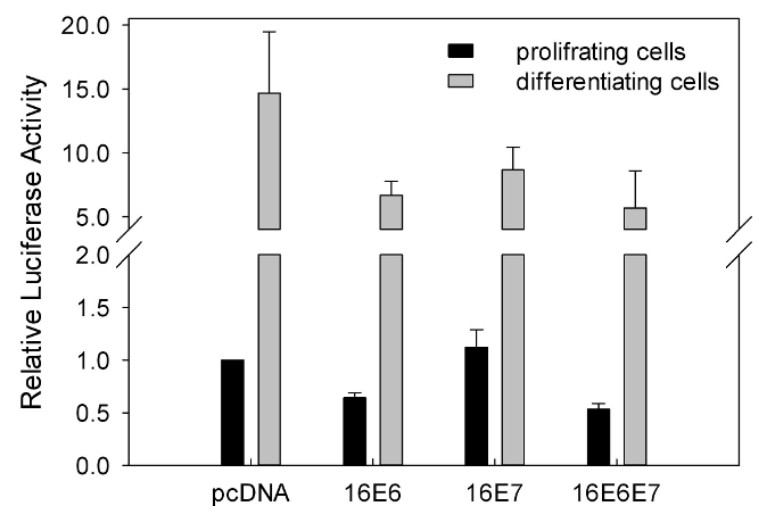

Figure 4 The effect of HPV oncogenes on the transcriptional activity of different promoters in HFK cells. (A) HFK cells were cOtransfected with $0.5 \mu \mathrm{g}$ of the p53 reporter construct p53-Luc, along with $0.25 \mu \mathrm{g}$ of either control vector (pcDNA) or $0.25 \mu \mathrm{g}$ of expression constructs encoding HPV16 E6 or E7 or both expression vectors $(0.125-0.125 \mu \mathrm{g})$ as indicated. (B) HFK cells were co-transfected with $0.5 \mu \mathrm{g}$ of the adenovirus E2 reporter construct pAdE2Luc, along with $0.25 \mu \mathrm{g}$ of either control vector (pcDNA) or $0.25 \mu \mathrm{g}$ of expression constructs encoding HPV16 E6 or E7 or both as indicated. (C) HFK cells were co-transfected with $0.5 \mu \mathrm{gg}$ of the IVL reporter construct pGL3-IVL, along with $0.25 \mu \mathrm{g}$ of either control vector (pcDNA) or $0.25 \mu \mathrm{g}$ of expression constructs encoding HPV16 E6 or E7 or both as indicated. After transfection, HFK cells were either left in serum-free medium or induced to differentiate in DMEM (with serum and high calcium). The luciferase activities are shown relative to the activity of cells co-transfected with reporter constructs and the empty expression vector (pcDNA). Data are from at least three independent experiments with standard errors shown as error bars. 


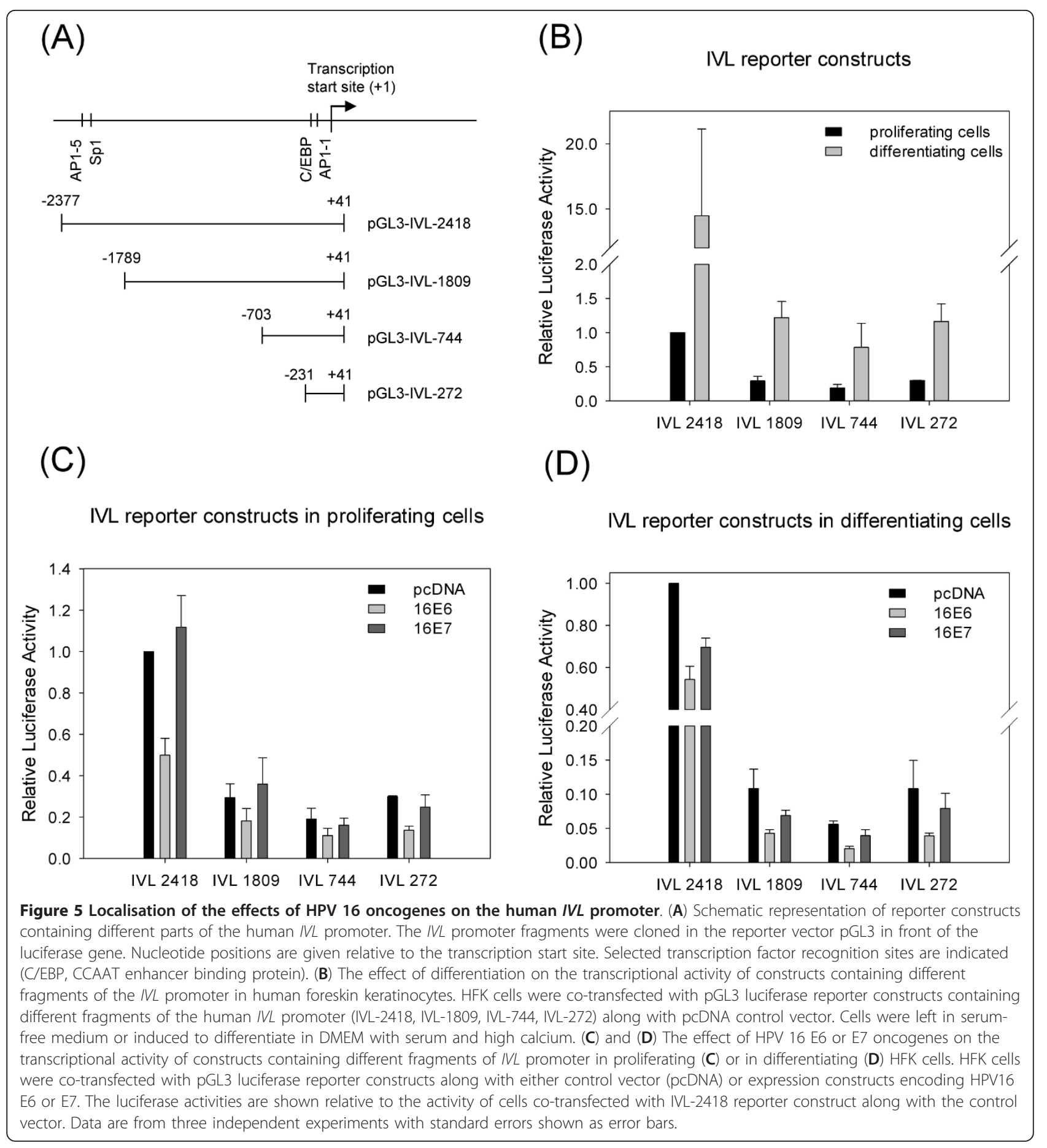

explore the molecular mechanisms behind gene expression alterations using luciferase reporter assays. We also found it important to study the effects of the HPV oncogenes on the expression of differentiation-regulated genes both in proliferating and in differentiating HFK cells as we thought that differentiating cells rather than proliferating cells reflect better the cellular environment required for the productive life cycle of HPV.
As expected, induction of differentiation of keratinocytes highly increased the endogenous mRNA levels of both $I V L$ and TG1 in HFK cells (Figure 2). Interestingly, the HPV 16 E6 and E7 oncogenes together had a very strong down-regulating effect on IVL mRNA but only a moderate effect on TG1 mRNA. This suggests that the HPV oncogenes may have different effects on the expression of different genes involved in the differentiation of 
squamous epithelial cells. Western blot analysis showed that the joint effect of HPV 16 E6 and E7 on transcriptional down-regulation resulted in excessive decrease of IVL protein levels as well, both in proliferating and in differentiating cells (Figure 3). In a previous study, the expression of HPV 6 or HPV 16 E7 was shown to result in a decrease of IVL protein levels in HFK cells [24]. We can conclude that the HPV 16 E6 and E7 oncogenes together seem to down-regulate basal IVL expression and also decrease the differentiation-induced expression of the $I V L$ gene in HFK cells.

The expression of genes involved in keratinocyte differentiation (including $I V L$ ) are generally regulated on the level of transcription [25]. Therefore, it seemed reasonable to investigate the effects of HPV 16 oncoproteins on $I V L$ promoter activity. This approach included transfecting HFK cells by HPV 16 E6 and/or E7 expression vectors along with luciferase reporter constructs containing the whole upstream-regulatory region (URR) of the human $I V L$ gene. In agreement with previous results [26], differentiation of HFK cells led to a significant increase in the transcriptional activity of the $I V L$ promoter. In proliferating HFK cells, HPV 16 E6, but not E7 caused a significant down-regulation of $I V L$ promoter activity. The HPV 16 E6 and E7 oncoproteins together caused a down-regulation of $I V L$ promoter activity in differentiating HFK cells (Figure 4). Taken together, these results suggest that the down-regulation of endogenous $I V L$ mRNA and protein levels in HFK cells by the HPV 16 E6 oncoprotein is caused by inhibition of IVL promoter activity. However, it can not be ruled out that HPV 16 E6 down-regulates the expression of $I V L$ or other differentiation-associated genes also by other mechanisms. For example, HPV 16 E6 was shown to down-regulate the expression of Notch1, which was suggested to have a role in the suppression of keratinocyte differentiation by E6 [27].

In order to localise the effect of the HPV oncogenes within the $I V L$ promoter, we made luciferase reporter constructs containing different parts of the URR of the human IVL gene. The URR of the human IVL gene contains a distal regulatory region (DRR, -2473/-1953 from transcription start site) and a proximal regulatory region (PRR, -241/-7 from the transcription start site) [26]. From the 5 possible AP1 (activator protein 1) binding sites in the URR, AP1-5 (in DRR) and AP1-1 (in PRR) are essential for optimal promoter activity [28]. AP1 factors (c-fos, fosB, Fra-1, Fra-2, c-jun, junB and junD) are expressed at specific epidermal layers and the expression pattern of these factors is thought to have a role in differentiation-regulated gene expression in keratinocytes $[25,29,30]$. Fra-1, junB and junD interact with AP1 sites within the human $I V L$ promoter and mediate phorbol ester responsiveness [28]. In our experiments, the level of inhibition by HPV 16 E6 was the highest for the construct containing the whole URR of the IVL gene, but an IVL reporter construct carrying only the PRR was still significantly inhibited by the HPV 16 E6 protein, both in proliferating and in differentiating HFK cells (Figure 5). This suggests that the PRR of $I V L$ gene contains binding sites for transcription factors that are regulated by HPV 16 E6.

HPV 16 E7 had a significant inhibitory effect only on the construct containing the full-length $I V L$ promoter (IVL 2418), and this effect was seen only in differentiating cells (Figure 5). This may suggest that the effect of E7 on the IVL promoter is less direct and/or less specific than that of E6. We find it conceivable that the effects of E7 seen on IVL expression (synergistic down-regulating effect with E6) and on IVL promoter (slight down-regulation only in differentiating cells) are caused not by a direct and specific interaction with the IVL promoter, but rather by recently described other mechanisms. For instance, the DEK protein was found to be transcriptionally up-regulated by HPV $16 \mathrm{E7}$, and this was shown to be important in the induction of cell proliferation and inhibition of the epithelial differentiation program [31,32]. Furthermore, nucleophosmin (NPM) was reported to be up-regulated by HPV 16 E7 at the posttranscriptional level, and this up-regulation was suggested to have a role in the inhibition of differentiation in keratinocytes [33].

Both the DRR and the PRR of the human IVL gene contains binding sites for AP1 transcription factors (PRR). The promoters of differentiation-associated keratinocyte genes usually contain binding sites for the AP1 factors, and these are thought to be important in the regulation of gene expression by differentiation stimuli $[25,29]$. It is also interesting to note that the HPV 16 E7 protein was shown to bind to AP1 transcription factors, including c-jun, junB, junD and c-fos [34]. Therefore, we suppose that the AP1 motifs in the promoter of the human $I V L$ gene may have a role in the regulation of gene expression by HPV oncoproteins. In order to prove this hypothesis, further research will be required using promoter mutagenesis and chromatin immunoprecipitation (ChIP) assays. It would be also interesting to study the effects of the HPV oncogenes on the expression of other genes (such as keratins, small prolin-rich proteins, S100 calcium binding proteins) involved in keratinocyte differentiation.

The decreased expression of IVL and other differentiation-regulated genes by the HPV oncoproteins may have an important role in the productive life cycle of the virus. HPV replication takes place in differentiating epithelial cells, which would exit the cell cycle in the absence of viral infection. The E7 oncogene is able to induce the progression of the cell cycle in differentiating 
keratinocytes, which is important for viral DNA replication $[6,7]$. On the other hand, the ability of the E6 oncogene to cause a delay in the induction of epithelial differentiation may also have a role in providing a cellular environment that is favourable for HPV replication. Our results indicate that one possible mechanism of the inhibition of keratinocyte differentiation by E6 may be the down-regulation of promoters of certain differentiation-regulated genes.

\section{Conclusions}

In this study, the human papillomavirus 16 (HPV 16) E6 and E7 oncogenes were found to have strong synergistic inhibitory effect on the expression of endogenous IVL mRNA and protein, both in replicating and in differentiating human foreskin keratinocyte (HFK) cells. In nondifferentiating HFK cells, HPV 16 E6 but not E7 downregulated the activity of the human $I V L$ promoter, and this effect could be localized to the proximal regulatory region (PRR) of the promoter. Our results indicate that the down-regulation of $I V L$ promoter activity by HPV 16 E6 significantly contribute to the inhibition of endogenous $I V L$ expression by the HPV 16 oncoproteins. In contrast, the down-regulation of endogenous IVL expression by HPV16 E7 is probably not caused by a direct and specific effect of E7 on the IVL promoter.

\section{Methods}

\section{Cell culture and retroviral transduction}

Primary human foreskin keratinocytes (HFK) were obtained from Invitrogen. HFK cells were cultured in Defined Keratinocyte-Serum Free Medium containing < $0.1 \mathrm{mM}$ calcium (DK-SFM, Invitrogen). PA317-LXSN, $-16 \mathrm{E} 6,-16 \mathrm{E} 7$, and $-16 \mathrm{E} 6 \mathrm{E} 7$ cells are recombinant retrovirus producing cell lines from the ATCC (American Type Culture Collection). These cell lines were maintained in Dulbecco's modified Eagle's medium (DMEM) supplemented with $10 \%$ foetal calf serum, $2 \mathrm{mM} \mathrm{L-glu-}$ tamine and antibiotics $(100 \mathrm{U} / \mathrm{ml}$ penicillin and $100 \mu \mathrm{g} /$ $\mathrm{ml}$ streptomycin). Primary keratinocytes were infected with culture supernatants from PA317 cell lines producing the control LXSN virus or LXSN-based retroviral vectors expressing HPV16 E6, HPV16 E7 or HPV16 E6/ E7 genes. These cells were selected in media containing G418 $(100 \mu \mathrm{g} / \mathrm{ml})$. Infected HFKs were either left untreated or induced to differentiate by culturing in DMEM (containing $1.8 \mathrm{mM}$ calcium and $10 \%$ foetal calf serum) for $24 \mathrm{~h}$.

\section{Plasmid constructs}

The luciferase reporter vector pGL3-IVL, containing a $3.7 \mathrm{~kb}$ fragment of the human $I V L$ gene regulatory region was described previously [35]. The other fragments of $I V L$ promoter were created by PCR using
pGL3-IVL as template and cloned into pGL3-Basic (Promega) between the XhoI and HindIII restriction sites resulting pGL3-IVL-2418, pGL3-IVL-1809, pGL3-IVL744 and pGL3-IVL-272 (Table 1). Amplifications were performed with GeneAmp High Fidelity System (Applied Biosystems) according to the manufacturer's protocol. The resulting clones were verified by sequencing. The p53-Luc reporter plasmid containing several copies of the p53 binding site was purchased from Agilent Technologies. The pAdE2Luc reporter construct containing the adenovirus E2 promoter was kindly provided by Dr. Ann Roman [36]. The pcDNA-16E6 and pcDNA-16E7 expression vectors were described previously [37].

\section{Transient transfection}

Primary human keratinocytes (within 3-6 passages) were plated on 6-well plates at approximately $80 \%$ confluence. The cells were co-transfected by $0.5 \mu \mathrm{g}$ of $I V L$ promoter-luciferase constructs (pGL3-IVL, pGL3-IVL-2418, pGL3-IVL-1809, pGL3-IVL-744 or pGL3-IVL-272) along with $0.25 \mu \mathrm{g}$ of expression vectors (pcDNA) encoding HPV 16 E6 and/or E7 genes using Effectene (Qiagen). The total amount of expression vectors was kept constant $(0.25 \mu \mathrm{g})$ in all transfection experiments. Transfection mix was added to cells in Opti-MEM (Invitrogen) and incubated for $5 \mathrm{~h}$ at $37^{\circ} \mathrm{C}$, after which the medium was changed to DK-SFM. Twenty-four hours after transfection, HFKs were either left untreated or induced to differentiate in DMEM (containing $1.8 \mathrm{mM}$ calcium and $10 \%$ foetal calf serum for $24 \mathrm{~h}$ ). The cells were washed with PBS (phosphate buffered saline) $48 \mathrm{~h}$ after transfection and lysed in Reporter Lysis Buffer (Promega). A Berthold luminometer and Luciferase assay system (from Promega) was used to measure luciferase activity. Bradford protein assay was performed to standardize for the protein concentration of the cell

Table 1 Sequences of PCR primers used to construct luciferase reporter vectors containing different fragments of the human IVL promoter

\begin{tabular}{|c|c|c|}
\hline $\begin{array}{l}\text { Plasmid } \\
\text { construct }\end{array}$ & Primer sequence $\left(5^{\prime}\right.$ to $\left.3^{\prime}\right)$ & $\begin{array}{l}\text { Restriction } \\
\text { site }\end{array}$ \\
\hline \multirow[t]{2}{*}{ pGL3-IVL-2418 } & GCGCCTCGAGCAGTGAAAGAACCTCTCCCA & Xhol \\
\hline & GCGCAAGCTTCCATCCGACACTTACCAGAC & HindIII \\
\hline \multirow[t]{2}{*}{ pGL3-IVL-1809 } & GCGCCTCGAGATGATCCAGGAACATGACAA & Xhol \\
\hline & GCGCAAGCTTCCATCCGACACTTACCAGAC & HindllI \\
\hline \multirow[t]{2}{*}{ pGL3-IVL-744 } & GCGCCTCGAGTTCTTATGAGCATGGCATTC & Xhol \\
\hline & GCGCAAGCTTCCATCCGACACTTACCAGAC & HindIII \\
\hline \multirow[t]{2}{*}{ pGL3-IVL-272 } & GCGCCTCGAGAGTTGAGCTACCAGAATCCT & Xhol \\
\hline & GCGCAAGCTTCCATCCGACACTTACCAGAC & HindIII \\
\hline
\end{tabular}

PCR products amplified using the indicated primers were digested by Xhol and HindIII and cloned into the luciferase reporter vector pGL3 
extracts. Each transfection experiment was performed independently at least three times.

\section{RT-PCR}

Total RNA was isolated from proliferating or differentiating transduced cells by using TRI reagent (Sigma). The High Capacity cDNA Reverse Transcription Kit (Applied Biosystems) was used to prepare cDNA. The PCR reaction was performed with GoTaq DNA polymerase (Promega) according to the manufacturer's protocol. The primer pairs used for amplifying HPV16 E6, E7 and GAPDH (glyceraldehyde 3-phosphate dehydrogenase) were previously described [38].

\section{Real-time RT-PCR}

After total RNA isolation, cDNA was synthesised as described above. The real-time PCR was performed on the 7500 Real Time PCR System (Applied Biosystems) using TaqMan Gene Expression Master Mix and Assays according to the manufacturer's recommendations (Applied Biosystems). The PCR amplification was carried out in a total volume of $20 \mu \mathrm{l}$. The TaqMan Gene Expression Assays used were for involucrin (IVL; Hs00846307_s1), transglutaminase 1 (TG1; Hs00165929_m1), cyclin-dependent kinase inhibitor 2A (CDKN2A, p16-INK4A; Hs00923894_m1), telomerase reverse transcriptase (TERT; Hs00162669_m1) and glyceraldehyde 3-phosphate dehydrogenase (GAPDH; 0711024) as endogenous control (Applied Biosystems). Each PCR reaction was performed in triplicate at least three times.

\section{Western blot}

Protein extract from proliferating or differentiating transduced HFK cells were prepared in RIPA lysis buffer (150 mM NaCl, 1\% NP-40, $50 \mathrm{mM}$ Tris- $\mathrm{HCl} \mathrm{pH} 8.0$, $0.5 \%$ Na-dezoxycholate, $0.1 \%$ SDS, $0.01 \%$ Na-azide, 1 mM EDTA, pH 7.4) supplemented with Complete EDTA-free Protease Inhibitor Cocktail (Roche). Cells were scraped, incubated on ice, and after centrifugation the supernatants were collected. The protein concentration of the lysates was measured by Bradford protein assay. Ten $\mu \mathrm{g}$ of protein extracts was electrophoresed through $10 \%$ polyacrilamide gels (SDS-PAGE) and electroblotted into nitrocellulose membrane. Membranes were blocked in Tris buffered saline-Tween (TBST: 10 $\mathrm{mM}$ Tris, $150 \mathrm{mM} \mathrm{NaCl}, 0.05 \%$ Tween 20, pH 7.9) containing 5\% non-fat dry milk. The blots were incubated with primary antibodies overnight at $4{ }^{\circ} \mathrm{C}$. The following primary antibodies were diluted 1:2000 in 5\% non-fat dry milk in TBST: mouse monoclonal anti-involucrin (sc-56555), mouse monoclonal anti-p53 (sc-126) from Santa Cruz Biotechnology and rabbit polyclonal antiactin (A2066) from Sigma. After washing in TBST, the membrane was incubated for $1 \mathrm{~h}$ with goat anti-mouse (sc-2005) or goat anti-rabbit (sc-2004) secondary antibodies conjugated with horseradish peroxidase (Santa Cruz Biotechnology) diluted 1:5000 in 5\% non-fat dry milk in TBST. Following washes in TBST, antibody complexes were visualized using the SuperSignal West Pico Chemiluminescent Substrate (Pierce) and exposed to X-ray films. The amounts of proteins were quantitatively determined by densitometry using Gel Doc 2000 gel documentation system (Bio-Rad) and the Quantity One (version 4.0.3) software. Protein levels of IVL and p53 were normalized to actin levels.

\section{Statistical analysis}

For the analysis of real-time RT-PCR results, the comparative $\mathrm{Ct}$ method was used to obtain the Relative Quantification (RQ) values with standard deviation and confidence intervals (7500 System SDS Software, version 1.4). To analyse the results of luciferase tests, mean and SEM (standard error of mean) of standardized luciferase values (from at least 3 independent experiments) were calculated, and the significance of differences between 2 mean values was evaluated using the 2 -sample $t$-test. Significance was accepted at $p<0.05$.

\section{Abbreviations}

AP1: activator protein 1; CCE: cornified cell envelope; CDKN2A: cyclindependent kinase inhibitor 2A; C/EBP: CCAAT enhancer binding protein; ChIP: chromatin immunoprecipitation; DK-SFM: Defined Keratinocyte-Serum Free Medium; DMEM: Dulbecco's modified Eagle's medium; DRR: distal regulatory region of the human involucrin gene; EDTA: ethylenediaminetetraacetic acid; GAPDH: glyceraldehyde 3-phosphate dehydrogenase; HFK: human foreskin keratinocyte; HPV: human papillomavirus; IVL: involucrin: NPM: nucleophosmin; P16INK4A: cyclindependent kinase inhibitor 2A protein; PBS: phosphate buffered saline; pRB: retinoblastoma protein; PRR: proximal regulatory region of the human involucrin gene; RQ: relative quantification; SDS: sodium dodecyl sulfate; SEM: standard error of mean; SPRR: small praline rich proteins; TBST: Tris buffered saline-Tween; TERT: telomerase reverse transcriptase; TG1: transglutaminase 1; URR: upstream regulatory region of the human involucrin gene.

\section{Acknowledgements}

We thank Dr. Daniel D. Bikle for the involucrin reporter construct and Dr. Ann Roman for the adenovirus E2 reporter construct. This study was supported by a grant from the Hungarian Scientific Research Fund (OTKA K 81422).

\section{Authors' contributions}

EG carried out the majority of experiments and participated in drafting the manuscript. AS participated in the optimization of the methods. AF took part in cloning the reporter constructs and optimisation of transfection. JK and LG participated in the design of the study and in data analysis. VG conceived and designed the study, produced the retrovirus vector transduced cell lines and helped to draft the manuscript. All authors have read and approved the final version of the manuscript.

\section{Competing interests}

The authors declare that they have no competing interests.

Received: 6 October 2011 Revised: 11 January 2012

Accepted: 14 February 2012 Published: 14 February 2012 


\section{References}

1. zur Hausen $\mathrm{H}$ : Papillomavirus infections-a major cause of human cancers. Biochim Biophys Acta 1996, 1288:F55-F78.

2. de Villiers EM, Fauquet C, Broker TR, Bernard HU, zur Hausen H: Classification of papillomaviruses. Virology 2004, 324:17-27.

3. Bosch FX, Lorincz A, Munoz N, Meijer CJ, Shah KV: The causal relation between human papillomavirus and cervical cancer. J Clin Pathol 2002, 55:244-265.

4. Munger K, Howley PM: Human papillomavirus immortalization and transformation functions. Virus Res 2002, 89:213-228.

5. Mantovani F, Banks L: The human papillomavirus E6 protein and its contribution to malignant progression. Oncogene 2001, 20:7874-7887.

6. Munger K, Basile JR, Duensing S, Eichten A, Gonzalez SL, Grace M, Zacny VL: Biological activities and molecular targets of the human papillomavirus E7 oncoprotein. Oncogene 2001, 20:7888-7898.

7. Longworth MS, Laimins LA: Pathogenesis of human papillomaviruses in differentiating epithelia. Microbiol Mol Biol Rev 2004, 68:362-372.

8. Cheng S, Schmidt-Grimminger DC, Murant T, Broker TR, Chow LT: Differentiation-dependent up-regulation of the human papillomavirus E7 gene reactivates cellular DNA replication in suprabasal differentiated keratinocytes. Genes Dev 1995, 9:2335-2349.

9. Jones $\mathrm{DL}$, Alani RM, Munger $\mathrm{K}$ : The human papillomavirus E7 oncoprotein can uncouple cellular differentiation and proliferation in human keratinocytes by abrogating p21Cip1-mediated inhibition of cdk2. Genes Dev 1997, 11:2101-2111.

10. Pei XF, Sherman L, Sun YH, Schlegel R: HPV-16 E7 protein bypasses keratinocyte growth inhibition by serum and calcium. Carcinogenesis 1998, 19:1481-1486.

11. Sherman L, Schlegel R: Serum- and calcium-induced differentiation of human keratinocytes is inhibited by the E6 oncoprotein of human papillomavirus type 16. J Virol 1996, 70:3269-3279.

12. Zehbe I, Richard C, DeCarlo CA, Shai A, Lambert PF, Lichtig H, Tommasino M, Sherman L: Human papillomavirus 16 E6 variants differ in their dysregulation of human keratinocyte differentiation and apoptosis. Virology 2009, 383:69-77.

13. Duffy CL, Phillips SL, Klingelhutz AJ: Microarray analysis identifies differentiation-associated genes regulated by human papillomavirus type 16 E6. Virology 2003, 314:196-205.

14. Lehr $E$, Brown DR: Infection with the oncogenic human papillomavirus type 59 alters protein components of the cornified cell envelope. Virology 2003, 309:53-60.

15. Lehr E, Hohl D, Huber M, Brown D: Infection with Human Papillomavirus alters expression of the small proline rich proteins 2 and 3. J Med Virol 2004, 72:478-483.

16. Eckert RL, Yaffe MB, Crish JF, Murthy S, Rorke EA, Welter JF: Involucrinstructure and role in envelope assembly. J Invest Dermatol 1993, 100:613-617.

17. Rose BR, Thompson CH, Tattersall MH, Elliott PM, Dalrymple C, Cossart YE: Identification of E6/E7 transcription patterns in HPV 16-positive cervical cancers using the reverse transcription/polymerase chain reaction. Gynecol Oncol 1995, 56:239-244

18. Howie HL, Katzenellenbogen RA, Galloway DA: Papillomavirus E6 proteins. Virology 2009, 384:324-334

19. Liu X, Roberts J, Dakic A, Zhang Y, Schlegel R: HPV E7 contributes to the telomerase activity of immortalized and tumorigenic cells and augments E6-induced hTERT promoter function. Virology 2008, 375:611-623.

20. Santin AD, Zhan F, Bignotti E, Siegel ER, Cane S, Bellone S, Palmieri M, Anfossi S, Thomas M, Burnett A, et al: Gene expression profiles of primary HPV16- and HPV18-infected early stage cervical cancers and normal cervical epithelium: identification of novel candidate molecular markers for cervical cancer diagnosis and therapy. Virology 2005, 331:269-291.

21. Wong YF, Cheung TH, Tsao GS, Lo KW, Yim SF, Wang WW, Heung MM, Chan SC, Chan LK, Ho TW, et al: Genome-wide gene expression profiling of cervical cancer in Hong Kong women by oligonucleotide microarray. Int J Cancer 2006, 118:2461-2469.

22. Kravchenko-Balasha N, Mizrachy-Schwartz S, Klein S, Levitzki A: Shift from apoptotic to necrotic cell death during human papillomavirus-induced transformation of keratinocytes. J Biol Chem 2009, 284:11717-11727.

23. Wan F, Miao X, Quraishi I, Kennedy V, Creek KE, Pirisi L: Gene expression changes during HPV-mediated carcinogenesis: a comparison between an in vitro cell model and cervical cancer. Int J Cancer 2008, 123:32-40.
24. Zhang B, Chen W, Roman A: The E7 proteins of low- and high-risk human papillomaviruses share the ability to target the pRB family member p130 for degradation. Proc Natl Acad Sci USA 2006, 103:437-442

25. Rossi A, Jang SI, Ceci R, Steinert PM, Markova NG: Effect of AP1 transcription factors on the regulation of transcription in normal human epidermal keratinocytes. J Invest Dermatol 1998, 110:34-40.

26. Eckert RL, Crish JF, Efimova T, Dashti SR, Deucher A, Bone F, Adhikary G, Huang G, Gopalakrishnan R, Balasubramanian S: Regulation of involucrin gene expression. J Invest Dermatol 2004, 123:13-22.

27. Yugawa T, Handa K, Narisawa-Saito M, Ohno S, Fujita M, Kiyono T: Regulation of Notch1 gene expression by p53 in epithelial cells. Mol Cell Biol 2007, 27:3732-3742.

28. Welter JF, Crish JF, Agarwal C, Eckert RL: Fos-related antigen (Fra-1), junB, and junD activate human involucrin promoter transcription by binding to proximal and distal AP1 sites to mediate phorbol ester effects on promoter activity. J Biol Chem 1995, 270:12614-12622.

29. Eckert RL, Crish JF, Banks EB, Welter JF: The epidermis: genes on-genes off. J Invest Dermatol 1997, 109:501-509.

30. Mehic D, Bakiri L, Ghannadan M, Wagner EF, Tschachler E: Fos and jun proteins are specifically expressed during differentiation of human keratinocytes. J Invest Dermatol 2005, 124:212-220.

31. Wise-Draper TM, Allen HV, Thobe MN, Jones EE, Habash KB, Munger K Wells SI: The human DEK proto-oncogene is a senescence inhibitor and an upregulated target of high-risk human papillomavirus E7. J Virol 2005, 79:14309-14317.

32. Wise-Draper TM, Mintz-Cole RA, Morris TA, Simpson DS, WikenheiserBrokamp KA, Currier MA, Cripe TP, Grosveld GC, Wells SI: Overexpression of the cellular DEK protein promotes epithelial transformation in vitro and in vivo. Cancer Res 2009, 69:1792-1799.

33. McCloskey R, Menges C, Friedman A, Patel D, McCance DJ: Human papillomavirus type 16 E6/E7 upregulation of nucleophosmin is important for proliferation and inhibition of differentiation. J Virol 2010, 84:5131-5139.

34. Antinore MJ, Birrer MJ, Patel D, Nader L, McCance DJ: The human papillomavirus type 16 E7 gene product interacts with and transactivates the AP1 family of transcription factors. EMBO J 1996 15:1950-1960.

35. Ng DC, Shafaee S, Lee D, Bikle DD: Requirement of an AP-1 site in the calcium response region of the involucrin promoter. J Biol Chem 2000 275:24080-24088.

36. Armstrong DJ, Roman A: The relative ability of human papillomavirus type 6 and human papillomavirus type 16 E7 proteins to transactivate E2F-responsive elements is promoter- and cell-dependent. Virology 1997, 239:238-246.

37. Murvai M, Borbély AA, Kónya J, Gergely L, Veress G: Effect of human papillomavirus type $16 \mathrm{E} 6$ and E7 oncogenes on the activity of the transforming growth factor-beta2 (TGF-beta2) promoter. Arch Virol 2004 149:2379-2392

38. Borbély AA, Murvai M, Kónya J, Beck Z, Gergely L, Li F, Veress G: Effects of human papillomavirus type 16 oncoproteins on survivin gene expression. J Gen Virol 2006, 87:287-294.

doi:10.1186/1743-422X-9-36

Cite this article as: Gyöngyösi et al:: Effects of human papillomavirus (HPV) type 16 oncoproteins on the expression of involucrin in human keratinocytes. Virology Journal 2012 9:36. 